\title{
Modeling Circuit Systems Coupled with Distributed Semiconductor Equations
}

\author{
Caren Tischendorf*
}

\begin{abstract}
For a refined network analysis, we are interested in circuit simulation including distributed models of semiconductors. We construct a mathematical model for nonlinear electric networks containing semiconductors described by the drift-diffusion equations. The focus lies on the coupling of the network DAEs and the semiconductor PDEs.

Furthermore, we study the behavior of the coupled systems with respect to time dependent perturbations using an index concept for abstract DAEs. We present a network topological criterion that guarantees index-1 systems.
\end{abstract}

\section{Introduction}

The miniaturization trend in the development of integrated circuits has reached a level where certain physical effects as, for example, parasitic capacities, temperature dependency and quantum effects cannot any longer be neglected. This implies a refined modeling of the devices which yields to comprehensive replacement circuits, usually called as compact models, for the circuit simulation.

The development of these compact models and the correct tuning of the big number of model parameters is nowadays so expensive that a direct coupling of circuit and device simulation becomes desirable. Naturally, a coupling of both simulations (see e.g. $[17,26,6,4]$ ) is very time consuming. However, in case of large circuits with a few critical semiconductor devices (used e.g. in RF applications), we expect that the simulation costs of such a coupled system become sufficiently low with regard to the short development cycles.

Aiming a development of fast and reliable simulations of the coupled systems, we start with constructing and analyzing the model equations. Regarding that the sensitivity of numerical solutions of DAEs with respect to perturbations depends strongly on the DAE index, we are interested in an adequate criterion for the coupled system. Therefore, we investigate the coupled system as abstract differential algebraic system (ADAS) and its index (see [13]). The ADAS index concept orientates on the tractability index for DAEs. It characterizes the sensitivity of the solution with respect to time dependent perturbations and it represents an extension of the time index introduced in [15] for time-dependent and nonlinear PDAEs. We neglect perturbations with respect to space here

\footnotetext{
* Supported by the DFG Research Center "Mathematics for key technologies" (FZT 86) in Berlin and by the German Federal Ministry of Education and Research (03TIM3B3).
} 
because, in circuit simulation, the main interest is devoted to the simulation of the transient behavior of the circuit.

Considering the results concerning the coupling of different circuit models [3], we can not expect that the index of the coupled system does not exceed the index of the sub-systems. Therefore, we focused our investigations to the question under which conditions the coupled system has index one, which means that the system is well-posed considering perturbations with respect to time. In [2], it has been shown that the index is one for network models including the stationary driftdiffusion equations if the network itself has index 1 and all semiconductors are connected by a capacitive path. Here, we consider network models including the instationary drift-diffusion equations and we are interested in necessary as well as sufficient criteria for index-1 systems.

The present paper is organized as follows. Section 2 introduces the network models described by a differential algebraic system. In Section 3, we describe the drift diffusion model for semiconductor elements. Section 4 is devoted to the modeling of the coupling of both systems. Finally, in Section 5, we consider the coupled system as abstract differential algebraic system and investigate the index as a measure for the behavior of the system with respect to time dependent perturbations.

\section{$2 \quad$ Network Modeling}

The numerical simulation of electric networks is closely connected to the network modeling. Circuit models have to meet two contradicting demands. On the one hand, they have to describe the physical behavior of the circuit as correct as possible. On the other hand, the models should be as simple as possible in order to reduce the computing time to a minimum.

A well established approach meeting both demands to a certain extent is the description of the network by the Modified Nodal Analysis (MNA). It bases on Kirchhoff's laws and the voltage-current relations of the network elements.

Introducing $j$ as the vector of all branch currents, $v$ as the vector of all branch voltages and $e$ as the vector of all node potentials (voltage with respect to the mass node), we may express Kirchhoff's current law (KCL) as

$$
A i=0,
$$

and Kirchhoff's voltage law (KCL) as

$$
v=A^{\mathrm{T}} e .
$$

The matrix $A$ represents the incidence matrix describing the node-to-branch relations. It is constant and has the entries $1,-1$ and 0 only. The voltagecurrent relation of basic network elements like resistances, capacitances and inductances may be described by

$$
I_{R}=g\left(v_{R}\right), \quad i_{C}=\frac{\mathrm{d} q_{C}}{\mathrm{~d} t}\left(v_{C}\right), \quad v_{L}=\frac{\mathrm{d} \phi_{L}}{\mathrm{~d} t}\left(i_{L}\right)
$$


respectively. For the description of more complex devices like transistors, the use of so-called compact models has been proven to be very efficient. The compact models represent replacement circuits of the device using basic network elements only. In Figure 1 you see a simple compact model for a metal-oxide-

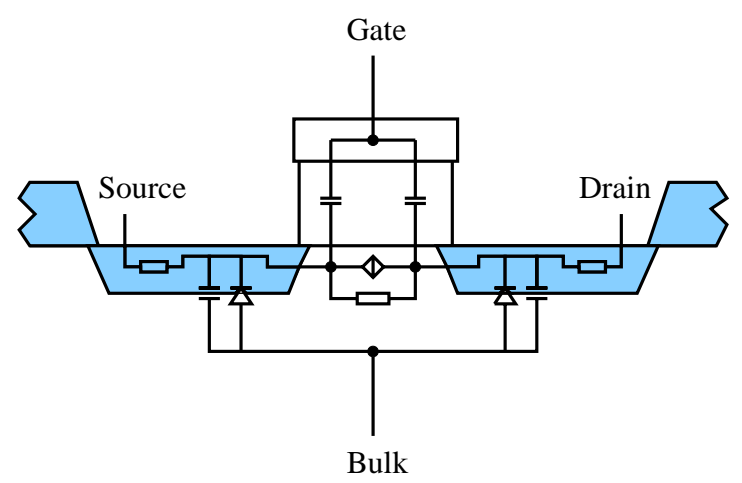

Figure 1: Compact model for a MOSFET

semiconductor field effect transistor (MOSFET).

Finally, the MNA leads in its charge oriented form to an equation system of the form

$$
\begin{aligned}
A_{C} \frac{\mathrm{d} q\left(A_{C}^{\mathrm{T}} e\right)}{\mathrm{d} t}+A_{R} g\left(A_{R}^{\mathrm{T}} e\right)+A_{L} j_{L}+A_{V} j_{V} & =-A_{I} i_{s}, \\
\frac{\mathrm{d} \phi\left(j_{L}\right)}{\mathrm{d} t}-A_{L}^{\mathrm{T}} e & =0, \\
A_{V}^{\mathrm{T}} e & =v_{s} .
\end{aligned}
$$

Here, $q$ and $\phi$ describe the charges and fluxes, respectively. $A_{C}$ describes the incidences of all capacitive branches. Correspondingly, $R, L, V$ and $I$ stand for resistances, inductances, voltage sources and current sources. The input functions $i_{S}$ and $v_{S}$ describe the current and voltage sources, respectively. For brevity, we consider only independent sources. This implies that $i_{S}$ and $v_{S}$ depend on time only. For a more detailed discussion of the MNA, we refer to [5].

The further miniaturization of the network elements leads to more and more complex compact models, which may contain more than 500 parameters today. The development of the compact models and the extraction of their parameters becomes so expensive that it is worth to consider the inclusion of original physical device models directly in the network simulation. The next section is devoted to the description of such models.

\section{Semiconductor Device Modeling}

Considering the literature, one finds an enormous amount of books and papers dealing with semiconductor device modeling. We want to mention here $[20,7$, 
$8,16,24]$. These contributions provide a comprehensive overview of the topic and focus onto the mathematical background.

Semiconductor device models describe the electron transport in the bulk of the semiconductor. In consideration of the degree of simplification one distinguishes between quantum level transport, semi-classical transport and balance equations. The first one yields the Schrödinger equation and the second one leads to the Boltzmann equation. Simplifying the Boltzmann equation further by the method of moments, we get the so called energy balance equations (considering four moments) or the drift diffusion equations (if we consider only two moments).

From the practical point of view, the interest in semiconductor device modeling is to replace as much laboratory testing as possible by numerical simulation in order to minimize the costs. This implies that the involved mathematical models cannot be too complicated. For most semiconductor technologies, the drift diffusion equations seem to represent a reasonable compromise between computational efficiency and an accurate description of the underlying physics.

However with the increased miniaturization of semiconductor devices, one comes closer and closer to the limits of validity of the drift diffusion equations. The reason for this is, on one hand, that in ever smaller devices the free carriers can not longer be modeled as a continuum. On the other hand, the drift diffusion equations are derived through a limiting process where the mean free path of a particle tends to zero. Through miniaturization this mean free path becomes larger and larger in comparison to the size of the device. In addition, quantum mechanical effects play a more an more important role in novel device structures.

But in spite of that the drift diffusion equations remain an important tool since the microscopic effects not described by them appear only locally. Thus, the most likely approach will be to use more sophisticated models only locally, and to use the drift diffusion equations in the parts of the device where they are sufficient to describe the physics (usually in the bulk of the semiconductor).

Therefore, we are concentrating here onto the drift diffusion equations considered as an important model description for the device part in coupled network and device simulation.

\subsection{The Drift Diffusion Model for Semiconductor Devices}

The model equations are given by the Poisson equation

$$
\operatorname{div}(\varepsilon \operatorname{grad} V)=q(n-p-N)
$$

for the electrostatic potential $V$ and the continuity equations

$$
\begin{aligned}
-\partial_{t} n+\frac{1}{q} \operatorname{div} J_{n} & =R\left(n, p, J_{n}, J_{p}, \operatorname{grad} V\right), \\
\partial_{t} p+\frac{1}{q} \operatorname{div} J_{p} & =-R\left(n, p, J_{n}, J_{p}, \operatorname{grad} V\right)
\end{aligned}
$$

for the electron and hole concentration, $n$ and $p$, respectively. The parameter $q$ denotes the elementary charge. Furthermore, $\varepsilon$ represents the material dependent dielectric constant. $J_{n}$ and $J_{p}$ describe the densities of the electron 
and hole current that is assumed to be a composition of a drift and a diffusion current. Consequently,

$$
\begin{aligned}
& J_{n}=q\left(D_{n} \operatorname{grad} n-\mu_{n} n \operatorname{grad} V\right), \\
& J_{p}=q\left(-D_{p} \operatorname{grad} p-\mu_{p} p \operatorname{grad} V\right) .
\end{aligned}
$$

Here, $q D_{n} \operatorname{grad} n$ describes the diffusion part of the electron current caused by the effort to achieve the chemical equilibrium. The drift part $-q \mu_{n} n \operatorname{grad} V$ is caused by the electric field by the effort to achieve the electrical equilibrium. The mobilities $\mu_{n}$ and $\mu_{p}$ as well as the diffusivities $\mathcal{D}_{n}$ and $D_{p}$ are bounded, strictly positive functions. They may depend on position $x$ (due to dependency on doping) and on the electrical field grad $V$. For a more detailed discussion of the model equations see e.g. [20, 21].

The system (6)-(10) represents a system of five coupled partial differential equations. The Poisson equation (6) is of elliptic type. Regarding the current density equations (9) and (10), the continuity equations (7) and (8) are of parabolic type.

Note that we assume a constant temperature. It is justified for applications with low performance devices. In case of high performance devices, one has to consider the temperature $T$ as a variable. The drift diffusion equations have to be completed by an energy balance equation (see e.g. $[25,12,1]$ ). It is a future task to combine such energy models with the network equations. In this paper, we shall concentrate on the network-device coupling using the classical instationary drift diffusion equations.

\subsection{The Boundary Conditions}

There are essentially three different types of materials a semiconductor is bounded by. The contacts between the network and the semiconductor are usually layers of metal. The second kind of bounding materials are insulators (e.g. oxide). Finally, they may be bounded by other semiconductors. Such semiconductorsemiconductor interfaces are called heterojunction. Here, we consider metal contacts only, since we restrict to the one-dimensional case for the first study. For a discussion of other boundary types, we refer to [19, 22].

Metal contacts are usually modeled as ohmic or Schottky contacts. Both types of contacts imply Dirichlet boundary conditions for the electrostatic potential $V$. Furthermore, we get Dirichlet boundary conditions for the electron and hole concentrations, $n$ and $p$, in case of Ohmic contacts. For Schottky contacts, we have Neumann boundary conditions for the electron and hole current densities, $J_{n}$ and $J_{p}$. For brevity and the sake of simplicity, we restrict to ohmic contacts.

Ohmic contacts are characterized by a high doping of the semiconductor. This implies a large band bending and a very thin barrier at the metal-semiconductor interface. In this case, tunneling of electrons is the dominant transport mechanism. It leads to high current densities at low voltage drops and, consequently, to a low resistance of the contact.

Since tunneling is not included in the drift-diffusion equations describing electron transport in the semiconductor volume, one should place the actual boundary for the simulation domain at the edge $x_{T}$, that means at the end of the tunneling region. At high doping concentrations, the tunneling length comprises 
the total depletion region, and the boundary is placed at the depletion layer edge. Consequently, we have charge neutrality at the actual boundary that means

$$
n-p-N=0 .
$$

Furthermore,

$$
V=V_{\text {ap }}+V_{\text {bi }},
$$

where $V_{\mathrm{ap}}$ is the applied voltage and $V_{\mathrm{bi}}$ is the so called builtin potential of the semiconductor. The builtin potential depends on semiconductor material, on doping concentration, and on temperature. Since we consider the temperature as constant, $V_{\mathrm{bi}}$ is a given function of the position variable $x$ only. The applied potential $V_{\text {ap }}$ depends on the node potentials $e$ at the contacts.

For very high doping (ideal ohmic contact), the resistance tends to zero which implies

$$
n p=n_{\mathrm{i}}^{2}
$$

with the intrinsic concentration $n_{i}$ depending on material and temperature. This leads to Dirichlet boundary conditions for the electron and hole concentrations

$$
\begin{array}{ll}
n=\frac{1}{2}\left(\sqrt{N^{2}+4 n_{i}^{2}}+N\right) & \text { on } \Gamma, \\
p=\frac{1}{2}\left(\sqrt{N^{2}+4 n_{i}^{2}}-N\right) & \text { on } \Gamma .
\end{array}
$$

Note, that the doping density $N$ and the intrinsic density $n_{\mathrm{i}}$ depend on the position $x$ only.

\section{Coupling of the Network and the Device Model Equations}

From the engineering point of view, the coupling of network and device simulation is not a new task (see e.g. $[4,18,26,14]$ ).

In contrast, the mathematical analysis of coupled network and device model equations represents a very young research field. First results have already been obtained in [9] and [8]. In [9], one semiconductor connected to a resistance has been considered. In [8] the connection of one semiconductor with a simple circuit has been studied. Simple means here that the currents entering the semiconductor may be expressed by a function of the applied voltages. In both cases, the network is treated as a special boundary condition for the semiconductor. This approach fails if more than one device belongs to the network. This is, in particular, the case for integrated circuits. However, the approach may be extended by a modification of the considered operator equation (see [22]).

More recently, networks containing uniform lossy transmission lines have been investigated in [11] and [10]. The resulting equation systems represent also coupled systems of differential algebraic equations and partial differential equations. In contrast to the case here, the PDEs are of hyperbolic type.

To the authors knowledge, an existence analysis for integrated networks containing semiconductor device models has only been developed in [2]. There, the 
stationary drift diffusion model for diodes have been considered. Here, we are dealing for the first time with the instationary case.

For brevity, we shall formulate the coupled system for a network that contains exactly one semiconductor. However, one can simply verify that the following arguments remain true if one considers a network with several semiconductors.

Considering the network equations (3)-(5), the task is now to include semiconductor devices that are described by the drift-diffusion model. Consequently, the currents of these semiconductor devices have to be added to the KCL equation (3). We denote the vector of all currents leaving semiconductor devices by $\hat{j}_{S}$. Let $b_{S}$ be the number of all these branch currents and let $n_{N}$ be the number of all nodes of the network. Then, we introduce the matrix $\hat{A}_{S} \in L\left(\mathbb{R}^{b_{S}}, \mathbb{R}^{n_{N}-1}\right)$ with the entries

$$
\hat{a}_{i k}:= \begin{cases}1 & \text { if the current } j_{S_{k}} \text { enters node } i, \\ 0 & \text { else. }\end{cases}
$$

This way, the matrix $\hat{A}_{S}$ describes the incidence of currents of semiconductor devices. However, it differs from the other incidence matrices $A_{E}$ ( $E$ corresponds to one of the basic network elements) by the fact that each column contains only entries 1 but no -1 . We arrive at

$$
A_{C} \frac{\mathrm{d} q}{\mathrm{~d} t}+A_{R} g\left(A_{R}^{\mathrm{T}} e\right)+A_{L} j_{L}+A_{V} j_{V}+\hat{A}_{S} \hat{j}_{S}=-A_{I} i_{s}
$$

instead of (3). Let $\Gamma_{k}$ be the $k$-th contact. Then, the current flowing through terminal $k$ is given by

$$
j_{S_{k}}=\int_{\Gamma_{k}} J_{t o t} \cdot \nu \mathrm{d} \sigma .
$$

$J_{\text {tot }}$ represents the total current density and $\nu$ is the outward unity normal vector at a point of $\Gamma_{k}$. In the semiconductor we meet three types of currents: the current of electrons, the current of holes, and the displacement current caused by the electrostatic potential. Consequently, the total current density is given by

$$
J_{t o t}=J_{n}+J_{p}-\varepsilon \operatorname{grad} \partial_{t} V .
$$

This implies

$$
\hat{j}_{S_{k}}=\int_{\Gamma_{k}}\left(J_{n}+J_{p}-\varepsilon \operatorname{grad} \partial_{t} V\right) \cdot \nu \mathrm{d} \sigma .
$$

Note that the displacement current disappears if one considers the stationary case only. But it can not be neglected in the non-stationary case in order to guarantee charge conservation (see next sub-section).

The semiconductor current represents only one part of the coupling. The other part is given by the boundary condition (11) for the electrostatic potential $V$

$$
V(x, t)=V_{\mathrm{ap}}(t)+V_{\mathrm{bi}}(x) \quad \text { on } \Gamma_{k} .
$$

$V_{\text {ap }}(t)$ is the applied potential at time $t$. It equals $e_{j}(t)$ if terminal $k$ (corresponding to $\Gamma_{k}$ ) enters node number $j$. Regarding equation (14), we find that

$$
V_{\mathrm{ap}}(t)= \begin{cases}0 & \text { if terminal } k \text { enters the mass node } \\ \hat{a}_{k}^{\mathrm{T}} e(t) & \text { else }\end{cases}
$$


for $\hat{a}_{k}^{\mathrm{T}}:=\left(\hat{a}_{1 k}, \ldots, \hat{a}_{n-1, k}\right)$. Introducing the vector $c(x)$ with the entries

$$
c_{k}(x)= \begin{cases}1 & \text { if } x \text { belongs to } \Gamma_{k}, \\ 0 & \text { else }\end{cases}
$$

we may write equation (17) as

$$
V(x, t)=c(x) \cdot \hat{A}_{S}^{\mathrm{T}} e(t)+W(x) \quad \text { on } \Gamma_{k} .
$$

\subsection{Charge Conservation and Homogenization}

Before we turn to the investigation of the coupled system as an abstract differential algebraic system, we want to reformulate the system as follows. First, we use the model property of charge conservation which allows the replacement of $\hat{A}_{S}$ by an incidence matrix $A_{S}$ with the same properties as $A_{C}$, etc. Secondly, we homogenize the boundary conditions for the potential $V$ in order to obtain function spaces that do not depend on the nodal potentials of the network.

The presented device model is charge conserving. In fact, we have

$$
\operatorname{div}\left(J_{n}+J_{p}\right)=q\left(\partial_{t} n-\partial_{t} p\right)
$$

if we add the continuity equations (7) and (8). Additionally,

$$
\operatorname{div}\left(\varepsilon \operatorname{grad} \frac{\partial}{\partial t} V\right)=q\left(\partial_{t} n-\partial_{t} p\right),
$$

if we differentiate the Poisson equation (6) with respect to time. This implies

$$
\operatorname{div} J_{t o t}=\operatorname{div}\left(J_{n}+J_{p}-\varepsilon \operatorname{grad} \partial_{t} V\right)=0
$$

and, by Gauss law,

$$
\frac{\mathrm{d} Q}{\mathrm{~d} t}=\oint_{\Gamma} J_{t o t} \cdot \nu \mathrm{d} \sigma=\int_{\Omega} \operatorname{div} J_{t o t} \mathrm{~d} x=0 .
$$

where $\Omega$ denotes the whole domain of the semiconductor. Furthermore, we get

$$
\begin{aligned}
\sum_{k} \hat{j}_{S_{k}}= & \sum_{\substack{k \\
\Gamma_{k} \subseteq \Gamma_{\mathrm{O}} \cup \Gamma_{\mathrm{S}}}} \int_{\Gamma_{k}}\left(J_{n}+J_{p}-\varepsilon \operatorname{grad} \partial_{t} V\right) \cdot \nu \mathrm{d} \sigma \\
& -\varepsilon \sum_{\substack{k \\
\Gamma_{k} \subseteq \Gamma_{\mathrm{MI}}}} \int_{\Gamma_{k}} \operatorname{grad} \partial_{t} V \cdot \nu \mathrm{d} \sigma=\oint_{\Gamma} J_{t o t} \cdot \nu \mathrm{d} \sigma=0,
\end{aligned}
$$

That means, that the current flowing through one terminal of the semiconductor may be described by the negative sum of the currents flowing through the other terminals. We choose one terminal (usual the bulk terminal) and call it the reference terminal. We delete the current of the reference terminal from $\hat{j}_{S}$ and denote the resulting vector by $j_{S}$. This implies

$$
\hat{A}_{S} \hat{j}_{S}=A_{S} j_{S}
$$


if the entries of the matrix $A_{S}$ are defined as

$$
a_{i k}:= \begin{cases}1 & \text { if the current } j_{S_{k}} \text { enters node } i \\ -1 & \text { if the reference terminal is connected to node } i \\ 0 & \text { else. }\end{cases}
$$

This way $A_{S}$ has the same form as the other incidence matrices $A_{C}$, etc. Including the row for the mass node, each column of $A_{S}$ has exactly one 1 and one -1 . Finally, we arrive at

$$
A_{C} C A_{C}^{\mathrm{T}} \frac{\mathrm{d}}{\mathrm{d} t} e+A_{R} G A_{R}^{\mathrm{T}} e+A_{L} j_{L}+A_{V} j_{V}+A_{S} j_{S}+A_{I} i_{s}=0
$$

instead of equation (15). Obviously, equation (16) is equivalent to

$$
j_{S_{k}}=\int_{\Gamma_{k}}\left(J_{n}+J_{p}-\varepsilon \operatorname{grad} \partial_{t} V\right) \cdot \nu \mathrm{d} \sigma
$$

Note that, in case of several semiconductors, one has to choose one reference terminal for each semiconductor and to follow the procedure for each semiconductor.

Considering existence results and Galerkin approaches for partial differential equations, Dirichlet boundary conditions are usually treated by a suitable choice of a function space as solution space. In our case, the Dirichlet boundary conditions (18) for the Poisson equation depend on the node potentials that are described only implicitly by the network equations. But it seems not comfortable to use a function space that is given implicitly only. Therefore, we shall homogenize the conditions (18) as follows.

From now we restrict to the one-dimensional case. Then, the semiconductor has only two boundary contacts. Without loss of generality, we choose $x=0$ as the left and $x=l$ as the right boundary, where $l$ is the length of the semiconductor. Let $f$ be a smooth function such that

$$
f(0)=1, \quad f(1)=0, \quad f^{\prime}(0)=0 .
$$

If the reference terminal enters node $e_{j}$, then we have

$$
c \cdot \hat{A}_{S}^{\mathrm{T}} e=e_{j}+f(x) A_{S}^{\mathrm{T}} e \quad \text { on } \Gamma \text {. }
$$

Introducing

$$
\tilde{V}(x, t):=V(x, t)-e_{j}(t)-f(x) \cdot A_{S}^{\mathrm{T}} e(t)-V_{\mathrm{bi}}(x),
$$

we get homogenous boundary conditions for $\tilde{V}$. Introducing

$$
\tilde{R}\left(n, p, J_{n}, J_{V}, \operatorname{grad} \tilde{V}\right):=R\left(n, p, J_{n}, J_{V}, \operatorname{grad} V\right)
$$

and summarizing all equations we arrive at the coupled system

$$
\begin{aligned}
A_{C} \frac{\mathrm{d} q\left(A_{C}^{\mathrm{T}} e\right)}{\mathrm{d} t}+A_{R} g\left(A_{R}^{\mathrm{T}} e\right)+A_{L} j_{L}+A_{V} j_{V}+A_{S} j_{S} & =-A_{I} i_{s}, \\
\frac{\mathrm{d} \phi\left(j_{L}\right)}{\mathrm{d} t}-A_{L}^{\mathrm{T}} e & =0 \\
A_{V}^{\mathrm{T}} e & =v_{s},
\end{aligned}
$$




$$
\begin{gathered}
\operatorname{div}(\varepsilon \operatorname{grad} \tilde{V})=q(n-p-N)-\operatorname{div}\left(\varepsilon \operatorname{grad}\left(f A_{S}^{\mathrm{T}} e+V_{\mathrm{bi}}\right)\right), \\
\partial_{t} n-\frac{1}{q} \operatorname{div} J_{n}=-\tilde{R}\left(n, p, J_{n}, J_{p}, \operatorname{grad} \tilde{V}\right), \\
\partial_{t} p+\frac{1}{q} \operatorname{div} J_{p}=-\tilde{R}\left(n, p, J_{n}, J_{p}, \operatorname{grad} \tilde{V}\right), \\
J_{n}=q\left(D_{n} \operatorname{grad} n-\mu_{n} n \operatorname{grad}\left(\tilde{V}+f A_{S}^{\mathrm{T}} e+V_{\mathrm{bi}}\right)\right), \\
J_{p}=q\left(-D_{p} \operatorname{grad} p-\mu_{p} p \operatorname{grad}\left(\tilde{V}+f A_{S}^{\mathrm{T}} e+V_{\mathrm{bi}}\right)\right), \\
j_{S}=\left[\left(J_{n}+J_{p}\right)-\varepsilon \operatorname{grad}\left(\partial_{t} \tilde{V}\right)\right]_{x=0}
\end{gathered}
$$

with the boundary conditions

$$
\tilde{V}=0, \quad n=\frac{1}{2}\left(\sqrt{N^{2}+4 n_{i}^{2}}+N\right), \quad p=\frac{1}{2}\left(\sqrt{N^{2}+4 n_{i}^{2}}-N\right) .
$$

\section{The ADAS Index of the Coupled System}

In order to treat coupled systems of partial differential equations and differentialalgebraic equations in a systematic way, abstract differential algebraic systems (ADASs) of the form

$$
\mathcal{A}(t) \frac{\mathrm{d}}{\mathrm{d} t}(\mathcal{D}(t) u(t))+\mathcal{B}(t) u(t)=q(t) \quad \text { for } t \in\left[t_{0}, T\right]
$$

have been investigated in [13]. This equation is to be understood as an operator equation with operators $\mathcal{A}(t), \mathcal{D}(t)$ and $\mathcal{B}(t)$ acting in real Hilbert spaces. More precisely, let $X, Y, Z$ and $\tilde{Z}$ be Hilbert spaces and

$$
\mathcal{A}(t): Z \rightarrow Y, \quad \mathcal{D}(t): X \rightarrow Z, \quad \mathcal{B}(t): X \rightarrow Y .
$$

As we will see later, we may formulate the coupled problem (23)-(32) as abstract DAEs with operators $\mathcal{A}, \mathcal{D}$ and $\mathcal{B}$ that do not explicitly depend on the time $t$. Therefore, we omit the argument $t$ for these operators in the following. Having the coupled problem in mind, we assume $\mathcal{A}$ to be linear.

From the finite dimensional case we know that the sensitivity of solutions of DAEs with respect to perturbations depends on its index. Since we are interested in the solution behavior of the coupled system, we want to follow the concept in [13] extending the tractability index to abstract DAEs. Therefore, we assume that the Fréchet derivatives $\mathcal{B}_{0}$ and $\mathcal{D}_{0}$ of the operators $\mathcal{B}$ and $\mathcal{D}$ exist. Furthermore, we introduce $\mathcal{G}_{0}:=\mathcal{A D}_{0}$.

Then, the abstract differential algebraic system

$$
\mathcal{A} \frac{\mathrm{d}}{\mathrm{d} t}(\mathcal{D} u(t))+\mathcal{B} u(t)=q(t) \quad \text { for } t \in\left[t_{0}, T\right]
$$

is called to have the index $\mu$ if

(i) $\operatorname{dim}\left(\operatorname{im} \mathcal{W}_{i}\right)>0$ for all $i=0, \ldots, \mu-1, \operatorname{dim}\left(\operatorname{im} \mathcal{W}_{\mu}\right)=0$ and

(ii) $\mathcal{G}_{\mu}$ is injective, 
where $\mathcal{W}_{i}$ and $\mathcal{G}_{i}$ are recursively defined as follows. $\mathcal{W}_{i}$ is a (bounded) projector with

$$
\operatorname{ker} \mathcal{W}_{i}=\overline{\operatorname{im\mathcal {G}}} .
$$

Furthermore,

$$
\mathcal{G}_{i+1}=\mathcal{G}_{i}+\mathcal{B}_{i} \mathcal{Q}_{i}, \quad \mathcal{B}_{i+1}=\mathcal{B}_{i} \mathcal{P}_{i}
$$

where $\mathcal{P}_{i}=\mathcal{I}-\mathcal{Q}_{i}$ and $\mathcal{Q}_{i}$ is a (bounded) projector with $\operatorname{im} \mathcal{Q}_{i}=\overline{\operatorname{ker} \mathcal{G}_{i}}$.

We want to note here, that the so defined index for an abstract differential algebraic system shall characterize the behavior of the system with respect to time-dependent perturbations. It should not be confused with the Fredholm index of operators.

Omitting the tilde notation for the potential $V$ of the coupled system (23)-(31) and regarding the boundary conditions (32), we are interested in solutions

$$
u(t)=\left(e(t), j_{L}(t), j_{V}(t), j_{S}(t), V(\cdot, t), n(\cdot, t), p(\cdot, t), J_{n}(\cdot, t), J_{p}(\cdot, t)\right)
$$

that belong to

$$
\begin{aligned}
X:= & \mathbb{R}^{n_{N}-1} \times \mathbb{R}^{n_{L}} \times \mathbb{R}^{n_{V}} \times \mathbb{R} \times\left(H^{2}(\Omega) \cap H_{0}^{1}(\Omega)\right) \times H^{1}(\Omega) \times H^{1}(\Omega) \times \\
& H(\operatorname{div} ; \Omega) \times H(\operatorname{div} ; \Omega) .
\end{aligned}
$$

for the coupled system (23)-(31). Here, $n_{N}, n_{L}$ and $n_{V}$ denote the number of nodes, the number of inductances and the number of voltage sources of the network, respectively. Recall that $\Omega=(0, l)$. We need the higher regularity for $V$ since we have to evaluate the gradient of $V$ at the boundary for the determination of the semiconductor current $j_{S}$. Furthermore, we choose

$$
\begin{aligned}
Y & :=\mathbb{R}^{n_{N}-1} \times \mathbb{R}^{n_{L}} \times \mathbb{R}^{n_{V}} \times\left(L_{2}(\Omega)\right)^{5} \times \mathbb{R}, \\
Z & :=\mathbb{R}^{n_{C}} \times \mathbb{R}^{n_{L}} \times \mathbb{R}^{n_{V}} \times \mathbb{R} \times\left(H^{1}(\Omega)\right)^{2},
\end{aligned}
$$

where $n_{C}$ denotes the number of capacities in the network. Note that the we have to choose $\left(L_{2}(\Omega)\right)^{5 n_{S}}$ and $\left(H^{1}(\Omega)\right)^{2 n_{S}}$ instead of $\left(L_{2}(\Omega)\right)^{5}$ and $\left(H^{1}(\Omega)\right)^{2}$, respectively, if the network contains not only one but $n_{S}$ semiconductors. Correspondingly, the space $X$ has also to be extended.

We may write the coupled system (23)-(31) as ADAS

$$
\mathcal{A} \frac{\mathrm{d}}{\mathrm{d} t}(\mathcal{D} u(t))+\mathcal{B} u(t)=q(t) \quad \text { for } t \in\left[t_{0}, T\right]
$$

with

$$
\mathcal{A}=\left(\begin{array}{ccccc}
A_{C} & 0 & 0 & 0 & 0 \\
0 & I & 0 & 0 & 0 \\
0 & 0 & 0 & 0 & 0 \\
0 & 0 & 0 & 0 & 0 \\
0 & 0 & 0 & 0 & 0 \\
0 & 0 & 0 & 0 & I \\
0 & 0 & 0 & 0 & 0 \\
0 & 0 & 0 & 0 & 0 \\
0 & 0 & 0 & 0 & 0
\end{array}\right), \quad \mathcal{D}(u)=\left(\begin{array}{c}
q\left(A_{C}^{\mathrm{T}} e\right) \\
\phi\left(j_{L}\right) \\
-\mathfrak{r} \varepsilon \operatorname{grad} V \\
n \\
p
\end{array}\right), \quad q=\left(\begin{array}{c}
-A_{I} i_{s} \\
0 \\
v_{s} \\
0 \\
0 \\
0 \\
0 \\
0 \\
0
\end{array}\right),
$$

and

$$
\mathcal{B}(u)=\left(\begin{array}{c}
A_{R} g\left(A_{R}^{\mathrm{T}} e\right)+A_{L} j_{L}+A_{V} j_{V}+A_{S} j_{S} \\
-A_{\mathrm{T}}^{\mathrm{T}} e \\
A_{V}^{\mathrm{T}} e \\
\operatorname{div}(\varepsilon \operatorname{grad} \tilde{V})-q(n-p-N)+\operatorname{div}\left(\varepsilon \operatorname{grad}\left(f A_{S}^{\mathrm{T}} e+V_{\mathrm{bi}}\right)\right) \\
-\frac{1}{q} \operatorname{div} J_{n}+\tilde{R}\left(n, p, J_{n}, J_{p}, \operatorname{grad} V\right) \\
\frac{1}{q} \operatorname{div} J_{p}+\tilde{R}\left(n, p, J_{n}, J_{p}, \operatorname{grad} V\right) \\
J_{n}-q\left(D_{n} \operatorname{grad} n-\mu_{n} n \operatorname{grad}\left(V+f A_{S}^{\mathrm{T}} e+V_{\mathrm{bi}}\right)\right) \\
J_{p}+q\left(-D_{p} \operatorname{grad} p-\mu_{p} p \operatorname{grad}\left(V+f A_{S}^{\mathrm{T}} e+V_{\mathrm{bi}}\right)\right) \\
j_{S}-\mathrm{r} J_{n}-\mathrm{r} J_{p}
\end{array}\right)
$$


where $\mathfrak{r}$ is the restriction operator onto the left boundary, i.e.

$$
\mathfrak{r} J(\cdot, t):=J(0, t) .
$$

From [23] we know that the network DAEs have an index $\leq 1$ if the network neither contains $L I$-cutsets nor CV-loops with at least one voltage source. LIcutsets are cutsets including inductances and current sources only. CV-loops are loops containing capacitances and voltage sources only. As we will see in the next theorem, LI-cutsets are also relevant for the coupled systems. However CV-loops have to be replaced by CVS-loops, i.e. loops consisting of capacitances, voltage sources and semiconductors.

Remark 5.1 The network does not contain LI-cutsets if and only if the matrix

$$
\left(A_{C}, A_{R}, A_{V}, A_{S}\right) \text { has full row rank. }
$$

The network does not contain CVS-loops with at least one voltage source or one semiconductor if and only if the matrix

$$
\left(Q_{C}^{T} A_{V}, Q_{C}^{T} A_{S}\right) \text { has full column rank }
$$

for any projector $Q_{C}$ onto $\operatorname{ker} A_{C}^{\mathrm{T}}$.

Furthermore, we will need the capacitance, resistance and inductance matrices

$$
C(v):=\frac{\mathrm{d} q(v)}{\mathrm{d} v}, \quad G(v):=\frac{\mathrm{d} g(v)}{\mathrm{d} v}, \quad \text { and } \quad L(j):=\frac{\mathrm{d} \phi(j)}{\mathrm{d} j},
$$

respectively.

Theorem 5.2 Let $C(v), G(v)$ and $L(j)$ be positive definite for all voltages $v$ and currents $j$. Then, the ADAS (35) has index 1 if and only if the network contains neither LI-cutsets nor CVS-loops with at least one voltage source or one semiconductor.

Proof: We start with the Fréchet derivatives

$$
\mathcal{D}_{0}(u)=\left(\begin{array}{cccccccccc}
C\left(A_{C}^{\mathrm{T}} e\right) A_{C}^{\mathrm{T}} e & 0 & 0 & 0 & 0 & 0 & 0 & 0 & 0 & 0 \\
0 & L\left(j_{L}\right) j_{L} & 0 & 0 & 0 & 0 & 0 & 0 & 0 & 0 \\
0 & 0 & 0 & 0 & -\mathfrak{r} \varepsilon \operatorname{crad} & 0 & 0 & 0 & 0 \\
0 & 0 & 0 & 0 & 0 & I & 0 & 0 & 0 \\
0 & 0 & 0 & 0 & 0 & 0 & I & 0 & 0
\end{array}\right)
$$

and

$$
\mathcal{B}_{0}(u)=\left(\begin{array}{ccccc}
A_{R} G\left(A_{R}^{\mathrm{T}} e\right) A_{R}^{\mathrm{T}} & A_{L} & A_{V} & A_{S} & 0 \\
-A_{L}^{\mathrm{T}} & 0 & 0 & 0 & 0 \\
A_{V}^{\mathrm{T}} & 0 & 0 & 0 & 0 \\
\varepsilon \Delta(f) A_{S}^{\mathrm{T}} & 0 & 0 & 0 & \varepsilon \Delta \\
0 & 0 & 0 & 0 & \tilde{R}_{V}(u) \operatorname{grad} \\
0 & 0 & 0 & 0 & \tilde{R}_{V}(u) \operatorname{grad} \\
q \mu_{n} n \operatorname{grad}(f) A_{S}^{\mathrm{T}} & 0 & 0 & 0 & q \mu_{n} n \operatorname{grad} \\
q \mu_{p} p \operatorname{grad}(f) A_{S}^{\mathrm{T}} & 0 & 0 & 0 & q \mu_{p} p \operatorname{grad} \\
0 & 0 & 0 & I & 0
\end{array}\right.
$$

$$
\left.\begin{array}{cccc}
0 & 0 & 0 & 0 \\
0 & 0 & 0 & 0 \\
0 & 0 & 0 & 0 \\
-q & q & 0 & \tilde{R}_{J_{p}}(u) \\
\tilde{R}_{n}(u) & \tilde{R}_{p}(u) & \tilde{R}_{J_{n}}(u)-\frac{1}{q} \text { div } & \tilde{R}(u) \\
\tilde{R}_{n}(u) & \tilde{R}_{p}(u) & \tilde{R}_{J_{n}}(u) & \tilde{R}_{J_{p}}(u)+\frac{1}{q} \text { div } \\
\mathrm{j}_{n}(u) & 0 & I & 0 \\
0 & \mathrm{j}_{p}(u) & 0 & I \\
0 & 0 & -\mathfrak{r} & -\mathfrak{r}
\end{array}\right)
$$


For brevity, we have introduced

$$
\begin{aligned}
& \mathfrak{j}_{n}(u):=q \mu_{n} \operatorname{grad}\left(V+f A_{S}^{\mathrm{T}} e+V_{\mathrm{bi}}\right)-q D_{n} \operatorname{grad}, \\
& \mathfrak{j}_{p}(u):=q \mu_{p} \operatorname{grad}\left(V+f A_{S}^{\mathrm{T}} e+V_{\mathrm{bi}}\right)+q D_{p} \operatorname{grad} .
\end{aligned}
$$

Then, the image space of

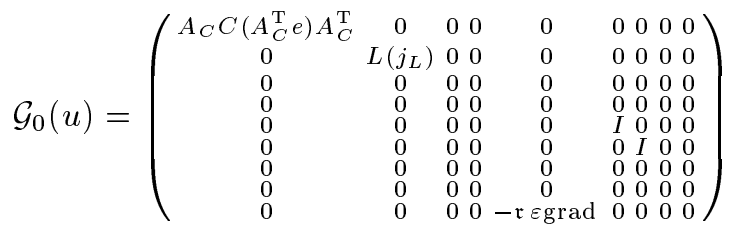

is given by

$$
\operatorname{im} A_{C}^{\mathrm{T}} \times \mathbb{R}^{n_{L}} \times 0 \times 0 \times H^{1}(\Omega) \times H^{1}(\Omega) \times 0 \times 0 \times \mathbb{R}
$$

Obviously,

$$
\mathcal{W}_{0}=\left(\begin{array}{ccccccc}
Q_{C}^{\mathrm{T}} & & & & & & \\
& 0 & & & & & \\
& & I & & & & \\
& & & I & & & \\
& & & 0 & & & \\
& & & & & & \\
& & & & & & \\
& & & & & & 0
\end{array}\right)
$$

is a projection operator along $\overline{\operatorname{im} \mathcal{G}_{0}(u)}$ for any projector $Q_{C}$ onto the nullspace of $A_{C}^{\mathrm{T}}$. Furthermore, $\mathcal{G}_{0}(u)$ has the nontrivial nullspace

$$
\operatorname{ker} A_{C}^{\mathrm{T}} \times 0 \times \mathbb{R}^{n_{V}} \times \mathbb{R}^{n_{S}} \times \mathcal{N}_{\mathrm{r} \operatorname{grad}} \times 0 \times 0 \times H^{1}(\Omega) \times H^{1}(\Omega)
$$

where

$$
\mathcal{N}_{\mathfrak{r} \operatorname{grad}}=\left\{v \in H^{2}(\Omega) \cap H_{0}^{1}(\Omega): \mathfrak{r} \operatorname{grad} v=0\right\}
$$

We choose

$$
\begin{aligned}
\mathcal{Q}_{\mathfrak{r g r a d}}: H^{2}(\Omega) \cap H_{0}^{1}(\Omega) & \rightarrow H^{2}(\Omega) \cap H_{0}^{1}(\Omega) \\
u & \mapsto v
\end{aligned}
$$

with

$$
v(x)=u(x)-\frac{x(l-x)}{l} \mathfrak{r} \operatorname{grad} u \quad \forall x \in \Omega
$$

as projection operator onto $\mathcal{N}_{\mathfrak{r} \text { grad }}$. Then,

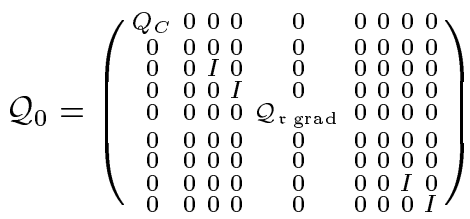


is a projection operator onto $\operatorname{ker} \mathcal{G}_{0}$. It yields

$$
\begin{aligned}
& \mathcal{G}_{1}(u)=\left(\begin{array}{cccc}
A_{C} C\left(A_{C}^{\mathrm{T}} e\right) A_{C}^{\mathrm{T}}+A_{R} G\left(A_{R}^{\mathrm{T}} e\right) A_{R}^{\mathrm{T}} Q_{C} & 0 & A_{V} & A_{S} \\
-A_{L}^{\mathrm{T}} Q_{C} & L\left(j_{L}\right) & 0 & 0 \\
A_{V}^{\mathrm{T}} Q_{C} & 0 & 0 & 0 \\
\varepsilon \Delta(f) A_{S}^{\mathrm{T}} Q_{C} & 0 & 0 & 0 \\
0 & 0 & 0 & 0 \\
0 & 0 & 0 & 0 \\
q \mu_{n} n \operatorname{grad}(f) A_{S}^{\mathrm{T}} Q_{C} & 0 & 0 & 0 \\
q \mu_{p} p \operatorname{grad}(f) A_{S}^{\mathrm{T}} Q_{C} & 0 & 0 & 0 \\
0 & 0 & 0 & I
\end{array}\right. \\
& \left.\begin{array}{ccccc}
0 & 0 & 0 & 0 & 0 \\
0 & 0 & 0 & 0 & 0 \\
0 & 0 & 0 & 0 & 0 \\
\varepsilon \Delta \mathcal{Q}_{\mathfrak{r} \operatorname{grad}} & 0 & 0 & 0 & 0 \\
\tilde{R}_{V}(u) \operatorname{grad} \mathcal{Q}_{\mathfrak{r} \operatorname{grad}} & I & 0 & \tilde{R}_{J_{n}}(u)-\frac{1}{q} \operatorname{div} & \tilde{R}_{J_{p}}(u) \\
\tilde{R}_{V}(u) \operatorname{grad} \mathcal{Q}_{\mathfrak{r} \operatorname{grad}} & 0 & I & \tilde{R}_{J_{n}}(u) & \tilde{R}_{J_{p}}(u)+\frac{1}{q} \operatorname{div} \\
q \mu_{n} n \operatorname{mrad} \mathcal{Q}_{\mathfrak{r} \operatorname{grad}} & 0 & 0 & I & 0 \\
q \mu_{p} p \operatorname{grad} \mathcal{Q}_{\mathfrak{r} \operatorname{grad}} & 0 & 0 & 0 & I \\
-\mathfrak{r} \varepsilon \operatorname{grad} & 0 & 0 & -\mathfrak{r} & -\mathfrak{r}
\end{array}\right)
\end{aligned}
$$

(ii) We show that $\mathcal{G}_{1}(u)$ is not injective if the network contains LI-cutsets or CVS-loops.

If the network contains an LI-cutset, then we find a nontrivial $w \in \mathbb{R}^{n_{N}-1}$ with

$$
A_{C}^{\mathrm{T}} w=0, A_{R}^{\mathrm{T}} w=0, A_{V}^{\mathrm{T}} w=0, A_{S}^{\mathrm{T}} w=0 .
$$

It implies $w=Q_{C} w$ and, consequently,

$$
v .=\left(w, L^{-1}\left(j_{L}\right) A_{L}^{\mathrm{T}} w, 0,0,0,0,0,0,0\right)
$$

belongs to the nullspace of $G_{1}(u)$.

If the network contains a CVS-loop, then we find a nontrivial $\left(w_{1}, w_{2}, w_{3}\right) \in$ $\mathbb{R}^{n_{C}} \times \mathbb{R}^{n_{V}} \times \mathbb{R}^{n_{S}}$ with

$$
A_{C} w_{1}+A_{V} w_{2}+A_{S} w_{3}=0 .
$$

Since $C(u)$ is positive definite, we find a $w_{4} \in \operatorname{im} P_{C}$ such that

$$
A_{C} w_{1}=A_{C} C\left(A_{C}^{\mathrm{T}} e\right) A_{C}^{\mathrm{T}} w_{4} .
$$

Choosing $w_{5} \in H^{2}(\Omega) \cap H_{0}^{1}(\Omega)$ with

$$
w_{5}(x)=\frac{x(l-x)}{\varepsilon l} w_{3}
$$

the vector function

$$
v .=\left(w_{4}, 0, w_{2}, w_{3}, w_{5}, 0,0,0,0\right)
$$

belongs to the nullspace of $G_{1}(u)$. The existence of at least one voltage source or one semiconductor in the CVS-loop ensures that the constructed $v$ is nontrivial because at least $w_{2}$ or $w_{3}$ is nontrivial.

(ii) Now we show that $\mathcal{G}_{1}(u)$ is injective if the network contains neither LIcutsets nor CVS-loops with at least one voltage source or one semiconductor. We assume that

$$
\mathcal{G}_{1}(u) w=0 .
$$


Integration of the 4 th line

$$
\varepsilon \Delta f A_{S}^{\mathrm{T}} Q_{C} w_{1}+\varepsilon \Delta Q_{\mathrm{r} \operatorname{grad}} w_{5}=0
$$

yields to

$$
\operatorname{grad} f A_{S}^{\mathrm{T}} Q_{C} w_{1}+\operatorname{grad} Q_{\mathfrak{r} \operatorname{grad}} w_{5}=\mathfrak{r} \operatorname{grad} f A_{S}^{\mathrm{T}} Q_{C} w_{1}=0,
$$

since $\mathfrak{r} \operatorname{grad} f=0$ for the chosen function $f$ (see (21)). Integrating once again, we obtain

$$
f A_{S}^{\mathrm{T}} Q_{C} w_{1}+Q_{\mathfrak{r g r a d}} w_{5}=\mathfrak{r} f A_{S}^{\mathrm{T}} Q_{C} w_{1}+\mathfrak{r} Q_{\mathfrak{r} \operatorname{grad}} w_{5}=A_{S}^{\mathrm{T}} Q_{C} w_{1},
$$

because $\mathfrak{r} f=1$ (cf. (21)) and $Q_{\mathfrak{r g r a d}} w_{5}$ belongs to $H_{0}^{1}(\Omega)$. Evaluating this equation at the point $x=l$, we arrive at

$$
0=A_{S}^{\mathrm{T}} Q_{C} w_{1} .
$$

From the 3 rd line of (38), we know that $A_{V}^{\mathrm{T}} Q_{C} w_{1}=0$. The 1 st line of (38) implies

$$
Q_{C}^{\mathrm{T}} A_{R} G(u) A_{R}^{\mathrm{T}} Q_{C} w_{1}+Q_{C}^{\mathrm{T}} A_{V} w_{3}+Q_{C}^{\mathrm{T}} A_{S} w_{4}=0 .
$$

Regarding the two relations for $w_{1}$ above and the fact that $G(u)$ is positive definite, we obtain

$$
\left(A_{C} A_{R} A_{V} A_{S}\right)^{\mathrm{T}} Q_{C} w_{1}=0, \quad \text { i.e. } \quad Q_{C} w_{1}=0,
$$

since the network does not contain LI-cutsets. Furthermore, using the 2nd and the 5th-8th line of $(38)$ as well as the fact that $L(u)$ is positive definite, we get

$$
w_{2}=0, \quad w_{8}=w_{9}=0, \quad w_{6}=w_{7}=0 .
$$

The 1st line of (38) reads as

$$
A_{C} C(u) A_{C}^{\mathrm{T}} w_{1}+A_{V} w_{3}+A_{S} w_{4}=0
$$

now and implies

$$
A_{C}^{\mathrm{T}} w_{1}=0, \quad w_{3}=0, \quad w_{4}=0,
$$

since $C(u)$ is positive definite and the network does not contain CVS-loops with at least one voltage source or one semiconductor. Together with (40) and the 9 th line of $(38)$, this yields to

$$
w_{1}=0, \quad \mathfrak{r} \operatorname{grad} w_{5}=0 .
$$

Regarding (39) and (40), we find

$$
Q_{\mathfrak{r} \operatorname{grad}} w_{5}=0
$$

and, consequently, $w_{5}=0$. That means that $G_{1}(u)$ is injective. 
(iii) It remains to show that $\overline{\operatorname{im} \mathcal{G}_{1}(u)}=Y$ if the network contains neither LI-cutsets nor CVS-loops with at least one voltage source or one semiconductor. Since $C^{\infty}(\Omega)$ is dense in $L_{2}(\Omega)$, it is enough to show that

$$
\mathbb{R}^{n-1} \times \mathbb{R}^{n_{L}} \times \mathbb{R}^{n_{V}} \times\left(C^{\infty}(\Omega)\right)^{5} \times \mathbb{R} \subseteq \operatorname{im} \mathcal{G}_{1}(u) .
$$

Let $y$ belong to $\mathbb{R}^{n-1} \times \mathbb{R}^{n_{L}} \times \mathbb{R}^{n_{V}} \times\left(C^{\infty}(\Omega)\right)^{5} \times \mathbb{R}$. With similar arguments like above, one can show that the matrix

$$
\left(\begin{array}{ccc}
A_{C} C(u) A_{C}^{\mathrm{T}}+A_{R} G(u) A_{R}^{\mathrm{T}} Q_{C} & A_{V} & A_{S} \\
A_{V}^{\mathrm{T}} Q_{C} & 0 & 0 \\
A_{S}^{\mathrm{T}} Q_{C} & 0 & 0
\end{array}\right)
$$

is nonsingular because the network contains neither LI-cutsets nor CVSloops with at least one voltage source or one semiconductor. Consequently, we find $w_{1}, w_{3}$ and $w_{4}$ such that

$$
\begin{aligned}
A_{C} C(u) A_{C}^{\mathrm{T}} w_{1}+A_{R} G(u) A_{R}^{\mathrm{T}} Q_{C} w_{1} & \\
+A_{V} w_{3}+A_{S} w_{4} & =y_{1} \\
A_{V}^{\mathrm{T}} Q_{C} w_{1} & =y_{3}, \\
A_{S}^{\mathrm{T}} Q_{C} w_{1} & =-\frac{1}{\varepsilon} \int_{0}^{l} \int_{0}^{\tau} y_{4}(s) \mathrm{d} s \mathrm{~d} \tau .
\end{aligned}
$$

Defining

$$
\begin{aligned}
& w_{2}= L^{-1}(u) y_{2}+L^{-1}(u) A_{L}^{\mathrm{T}} Q_{C} w_{1}, \\
& w_{5}(x)= \frac{1}{\varepsilon} \int_{0}^{x} \int_{0}^{\tau} y_{4}(s) \mathrm{d} s \mathrm{~d} \tau+(1-f(x)) A_{S}^{\mathrm{T}} Q_{C} w_{1} \\
& \quad \quad+\frac{x(l-x)}{\varepsilon l}\left(w_{4}-y_{9}-\mathfrak{r} y_{7}-\mathfrak{r} y_{8}\right), \\
& w_{8}= y_{7}-\frac{q}{\varepsilon} \mu_{n} n \int_{0}^{x} y_{4}(s) \mathrm{d} s, \\
& w_{9}= y_{8}-\frac{q}{\varepsilon} \mu_{p} p \int_{0}^{x} y_{4}(s) \mathrm{d} s, \\
& w_{6}= y_{5}-\tilde{R}_{V}(u) \operatorname{grad} Q_{\mathfrak{r g r a d}} w_{5}-\tilde{R}_{J_{n}}(u) w_{8}+\frac{1}{q} \operatorname{div} w_{8}-\tilde{R}_{J_{p}}(u) w_{9}, \\
& w_{7}=y_{6}-\tilde{R}_{V}(u) \operatorname{grad} Q_{\mathfrak{r g r a d}} w_{5}-\tilde{R}_{J_{n}}(u) w_{8}-\tilde{R}_{J_{p}}(u) w_{9}-\frac{1}{q} \operatorname{div} w_{9}
\end{aligned}
$$

successively, we obtain $w \in X$ and $\mathcal{G}_{1}(u) w=y$. Note that (42) ensures that $w_{5}(l)=0$ in order to guarantee $w_{5} \in H_{0}^{1}(\Omega)$.

\section{Conclusions}

The further miniaturization demands a refined network analysis describing certain semiconductor elements by contributed models. Using the instationary drift-diffusion model, the device equations represent a system of elliptic and parabolic differential equations. The network is described by a differentialalgebraic system. Both systems are mutually coupled via boundary conditions.

The coupled system can be analyzed as abstract differential algebraic system. For the one-dimensional case (with respect to position), network topological 
criteria are described that guarantee index- 1 systems. It is still an open question for the higher-dimensional case. Furthermore, it will be of interest under which conditions the coupled system has index 2 .

The presented index results form a basis for the discussion of suitable discretizations of the coupled system. Following the method of lines one should look for space discretizations that imply a DAE with the same index as the abstract DAE has.

\section{Acknowledgments}

The author wishes to thank Prof. R. März, Prof. G. Wachtuka, Dr. U. Feldmann, Dr. M. Günther, Dr. R. Lamour and A. Bartel for very fruitful discussions.

\section{References}

[1] G. Albinus, H. Gajewski, and R. Hünlich. Thermodynamic design of energy models of semiconductor devices. Nonlinearity, 15(2):367-383, 2002.

[2] G. Alì, A. Bartel, M. Günther, and C. Tischendorf. Elliptic partial differential-algebraic multiphysics models in electrical network design. Technical Report 02/05, Institute of Scientific Computing and Mathematical Modeling, University of Karlsruhe, 2002. To appear in Math. Models Meth. Appl. Sci. 2003.

[3] M. Arnold and M. Günther. Preconditioned dynamic iteration for coupled differential-algebraic systems. BIT Numerical Mathematics, 41:1-25, 2001.

[4] W. L. Engl, R. Laur, and H. K. Dirks. MEDUSA - A simulator for modular circuits. IEEE Trans. CAD, 1(2):85-93, 1982.

[5] D. Estévez Schwarz and C. Tischendorf. Structural analysis of electric circuits and consequences for MNA. Int. J. Circ. Theor. Appl., 28:131162,2000 .

[6] H. Gajewski. Analysis und Numerik des Ladungstransports in Halbleitern. GAMM Mitteilungen, 16:35-57, 1993.

[7] H. Gajewski and K. Gröger. On the basic equations for carrier transport in semiconductors. J. Math. Anal. Appl., 113:12-35, 1986.

[8] H. Gajewski and K. Gröger. Semiconductor equations for variable mobilities based on Boltzmann statistics or Fermi-Dirac statistics. Math. Nachr., 140:7-36, 1989.

[9] K. Gröger. Initial boundary value problems from semiconductor device theory. ZAMM, 67(8):345-355, 1987.

[10] M. Günther. Partielle differential-algebraische Systeme in der numerischen Zeitbereichsanalyse elektrischer Schaltungen. Number 343 in FortschrittBerichte VDI, Reihe 20, Rechnerunterstützte Verfahren. VDI Verlag, Düsseldorf, 2001. Habilitation. 
[11] M. Günther. A PDAE model for interconnected linear RLC networks. Mathematical and Computer Modelling of Dynamical Systems, 7(2):189203, 2001.

[12] A. Jüngel. Quasi-hydrodynamic Semiconductor Equations. Birkhäuser, Basel, 2001.

[13] R. Lamour, R. März, and C. Tischendorf. PDAEs and further mixed systems as abstract differential algebraic systems. Technical Report 01-11, Institute of Mathematics, Humboldt-Univ. of Berlin, 2001.

[14] J. Litsios, B. Schmithüsen, U. Krumbein, A. Schenk, E. Lyumkis, B. Polsky, and W. Fichtner. DESSIS 3.0 Manual. ISE Integrated Systems Engeneering, Zürich, 1996.

[15] W. Lucht, K. Strehmel, and C. Eichler-Liebenow. Indexes and special discretization methods for linear partial differential algebraic equations. BIT, 39(3):484-512, 1999.

[16] P. A. Markowich, C. A. Ringhofer, and C. Schmeiser. Semiconductor equations. Springer Verlag Wien, 1990.

[17] F.M. Rotella. Mixed circuit and device simulation for analysis, design, and optimization of opto-electronic, radio frequency, and high speed semiconductor devices. PhD thesis, Stanford University, 2000.

[18] S. Scharfenberg. Mixed-Level Circuit-Device Simulation auf einem heterogenen Workstation-Cluster. Shaker Verlag, 1996. PhD thesis.

[19] D. Schroeder. Modelling of Interface Carrier Transport for Device Simulation. Computational Microelectronics. Springer-Verlag, Wien New York, 1990.

[20] S. Selberherr. Analysis and Simulation of Semiconductor Devices. SpringerVerlag, Wien New York, 1984.

[21] S. M. Sze. Physics of Semiconductor Devices. John Wiley \& Sons, New York, 1981.

[22] C. Tischendorf. Modeling and numerical analysis of coupled systems of partial and differential algebraic equations in circuit and device simulation. In preparation.

[23] C. Tischendorf. Topological index calculation of DAEs in circuit simulation. Surv. Math. Ind., 8:187-199, 1999.

[24] G. Wachutka. Unified framework for thermal, electrical, magnetic, and optical semiconductor device modeling. COMPEL, 5:311-321, 1991.

[25] G. Wachutka. Consistent treatment of carrier emission and capture kinetics in electrothermal and energy transport models. Microelectronics Journal, 26:307-315, 1995.

[26] C. Wasshuber, H. Kosina, and S. Selberherr. SIMON - A simulator for single-electron tunnel devices and circuits. IEEE CAD, 16(9):937-944, 1997. 